\title{
PAVING SMALL MATRICES AND THE KADISON-SINGER EXTENSION PROBLEM
}

\author{
GARY WEISS AND VREJ ZARIKIAN
}

Abstract. We compute paving parameters for classes of small matrices and the matrices that yield them. The convergence to 1 or not of the sequence of these parameters is equivalent to the Kadison-Singer Extension Problem.

Mathematics subject classification (2010): Primary: 46L30; secondary: 15A60.

Keywords and phrases: Kadison-Singer extension problem, Anderson's paving problem.

\section{REFERENCES}

[1] C. Akemann, N. Weaver, $B(H)$ has a pure state which is not multiplicative on any masa, Proc. Natl. Acad. Sci. USA, 105, 14 (2008), 5313-5314 (electronic).

[2] J. Anderson, Extensions, restrictions, and representations of states on $C^{*}$-algebras, Trans. Amer. Math. Soc., 249, 2 (1979), 303-329.

[3] J. ANDERSON, Extreme points in sets of positive linear maps on $B(H)$, J. Funct. Anal., 31, 2 (1979), 195-217.

[4] K. Berman, H. Halpern, V. Kaftal, G. Weiss, Matrix norm inequalities and the relative Dixmier property, Integral Equations Operator Theory, 11, 1 (1988), 28-48.

[5] P. Casazza, D. Edidin, D. Kalra, V. I. Paulsen, Projections and the Kadison-Singer problem, Oper. Matrices, 1, 3 (2007), 391-408.

[6] P. J. Davis, Circulant matrices, A Wiley-Interscience Publication. Pure and Applied Mathematics. John Wiley \& Sons, New York-Chichester-Brisbane, 1979. xv+250 pp.

[7] H. Halpern, V. Kaftal, G. Weiss, Matrix pavings in $B(H)$, Operators in indefinite metric spaces, scattering theory and other topics (Bucharest, 1985), 201-214, Oper. Theory Adv. Appl., 24, Birkhuser, Basel, 1987.

[8] R. V. Kadison, I. M. Singer, Extensions of pure states, Amer. J. Math., 81 (1959), 383-400.

[9] R. MathiAs, The spectral norm of a nonnegative matrix, Linear Algebra Appl., 139 (1990), 269-284.

[10] V. I. Paulsen, M. Raghupathi, Some new equivalences of Anderson's paving conjectures, Proc. Amer. Math. Soc., 136, 12 (2008), 4275-4282.

[11] R. C. Read, R. J. Wilson, An atlas of graphs, Oxford Science Publications. The Clarendon Press, Oxford University Press, New York, 1998. xii+454 pp. 